\title{
Hypoxia-associated markers in gastric carcinogenesis and HIF-2 $\alpha$ in gastric and gastro-oesophageal cancer prognosis
}

\author{
EA Griffiths ',2, SA Pritchard ${ }^{3}$, SM McGrath ${ }^{3}$, HR Valentine', PM Price', IM Welch ${ }^{2}$ and CML West*, \\ 'Academic Department of Radiation Oncology, School of Cancer \& Imaging Sciences, The University of Manchester, Christie Hospital, Wilmslow Road, \\ Withington, Manchester M20 4BX, UK; ${ }^{2}$ Department of Gastrointestinal Surgery, South Manchester University Hospitals NHS Trust, Manchester M23 \\ 9LT, UK: ${ }^{3}$ Department of Histopathology, South Manchester University Hospitals NHS Trust, Manchester M23 9LT, UK
}

The study investigated hypoxia-associated markers (HIF-2 $\alpha$, Epo, Epo-R, Glut-I and VEGF) along with Ki-67 in a gastric carcinogenesis model, and the prognostic significance of hypoxia-inducible factor (HIF)-2 $\alpha$ in surgically treated gastro-oesophageal cancer. Protein expression was examined using immunohistochemistry on formalin-fixed, paraffin-embedded biopsies of normal mucosa ( $n=20$ ), Helicobacter pylori-associated gastritis $(n=24)$, intestinal metaplasia $(n=24)$, dysplasia $(n=12)$ and intestinal $(n=19)$ and diffuse $(n=2 \mathrm{I})$ adenocarcinoma. Relationships between HIF-2 $\alpha$ expression and prognosis were assessed in resection specimens from I77 patients with gastric and gastro-oesophageal junction adenocarcinoma. Expression of all markers increased with progression along the gastric carcinogenesis sequence $(P=0.000 \mathrm{I})$. Hypoxia-inducible factor- $2 \alpha$ was expressed in $63 \%$ of 177 resection specimens and at a high level in 44\%. The median overall survival in patients with HIF-2 $\alpha$-expressing tumours was 22 (95\% Cl I8-26) months, whereas those with HIF-2 $\alpha$-negative tumours had a median survival of 37 ( $95 \% \mathrm{Cl} 29-44)$ months $(P=0.0$ I5). Hypoxia-inducible factor- $2 \alpha$ had no independent prognostic significance in multivariate analysis. In view of the lack of independent prognostic significance, HIF- $2 \alpha$ has no role as a routine prognostic indicator. However, the high expression of HIF- $2 \alpha$ suggests that it may be of value as a potential therapeutic target.

British Journal of Cancer (2008) 98,965-973. doi:10.1038/sj.bjc.6604210 www.bjcancer.com

Published online 19 February 2008

(c) 2008 Cancer Research UK

Keywords: gastric cancer; gastro-oesophageal junction tumours; HIF-2 $\alpha$; hypoxia; carcinogenesis

Gastric and oesophageal cancers are among the most common malignancies worldwide and contribute significantly to global cancer mortality (Parkin et al, 2001). To improve the diagnosis and prognosis of gastric and gastro-oesophageal cancer, it is important to fully understand the molecular mechanisms underlying carcinogenesis. The well-described premalignant sequences of these tumour types make them ideal to study and explore potential molecular mechanisms of carcinogenesis by immunohistochemistry. Histologically, gastric cancers can be classified into two types: diffuse and intestinal. For the development of intestinal gastric cancer, a multistep process involving a progressive cascade of molecular and morphological changes has been proposed by Correa (2004). Diffuse tumours have no known premalignant precursor lesions. For both types of tumour, the carcinogenesis process is believed to be initiated by Helicobacter pylori infection and the risk of gastric cancer development has been related to H. pylori strain type, other environmental factors, host genetic factors and immune-related polymorphisms (Nardone et al, 2004).

Tumour hypoxia is a key factor driving the development of malignancy, and the master regulatory protein in the response of cells to changing oxygen levels is the hypoxia-inducible factor

\footnotetext{
* Correspondence: Dr CML West, Academic Radiation Oncology, The University of Manchester, Christie Hospital, Wilmslow Road, Withington, Manchester, M20 4BX, UK;

E-mail: Catharine.West@manchester.ac.uk

Received 4 October 2007; revised 3 January 2008; accepted 3 January 2008; published online 19 February 2008
}

(HIF). Researchers have hypothesised that hypoxia plays a primary role in the carcinogenesis process. Studies have shown progressively increased HIF- $1 \alpha$ expression in breast (Bos et al, 2001), skin (Costa et al, 2001) and cervical (Acs et al, 2003) cancer development. More recently, we have shown increased expression of HIF- $1 \alpha$ in gastric cancer development (Griffiths et al, 2007b) and in adenocarcinoma $v s$ dysplasia in the Barrett's oesophageal cancer sequence (Griffiths et al, 2007a). Hypoxia-inducible factor$1 \alpha$ is a key mediator of transcription and upregulates genes involved in a variety of processes: vascular endothelial growth factor (VEGF); erythropoietin (Epo) and its receptor (Epo-R), which regulates erythropoiesis by stimulating the growth and differentiation of red blood cell precursors (Yasuda et al, 2003; Ratcliffe, 2007). Erythropoietin and its receptor (Epo-R) are expressed in a number of cancers and are involved in breast (Acs et al, 2002), endometrial (Acs et al, 2004), melanoma (Kumar et al, 2005) and prostate (Feldman et al, 2006) tumorigenesis.

Recently, a number of proteins have been identified that are closely related to HIF- $1 \alpha$ and control the transcription of hypoxiaregulated genes in a similar way (HIF- $2 \alpha$ and HIF- $3 \alpha$ ) (Calzada and del Peso, 2007). A study in non-small cell lung cancer showed that HIF- $2 \alpha$ expression was related to a poor outcome whereas HIF- $1 \alpha$ expression was not (Giatromanolaki et al, 2001). Another study showed a predominant role of HIF- $2 \alpha$ over HIF- $1 \alpha$ in the regulation of the transcriptional response to hypoxia in renal cell carcinoma (Sowter et al, 2003). These findings raise the possibility of tissue-specific differences in the relative importance of HIF proteins in determining tumour progression and prognosis. We 
have previously shown that HIF- $1 \alpha$ is involved in gastric carcinogenesis and invasive edge tumour expression is associated with an adverse prognosis (Griffiths et al, 2007b). Hypoxiainducible factor- $2 \alpha$ expression, however, has not yet been assessed as a prognostic marker in gastro-oesophageal cancer.

The hypothesis underlying the research is that hypoxia plays a role in the aetiology and prognosis of gastro-oesophageal cancer. The specific goals of the research were to investigate whether the expression of hypoxia-associated proteins increases along the gastric carcinogenic sequence. Three hypoxia-associated markers were selected that have not been assessed in gastric carcinogenesis: HIF- $2 \alpha$, Epo and Epo-R. Although studied previously, VEGF and Glut-1 were also studied. The widely investigated Ki-67 was included as a comparator. The expression of the proteins was assessed using immunohistochemistry in paraffin-embedded material representing the gastric carcinogenesis sequence. A further aim was to assess the prognostic value of HIF- $2 \alpha$ expression in surgically treated gastric and gastro-oesophageal cancer patients.

\section{PATIENTS AND METHODS}

\section{Tissue specimens}

The study was approved by the South Manchester Ethics Committee. Tissues were obtained from the Department of Histopathology, South Manchester University Hospitals NHS Trust. Formalin-fixed endoscopic gastric biopsy samples obtained were of normal gastric mucosa $(n=20), H$. pylori-associated gastritis $(n=20)$, intestinal metaplasia $(n=20)$, epithelial dysplasia $(n=12)$ and intestinal $(n=19)$ and diffuse $(n=21)$ gastric adenocarcinoma. Four of the biopsies had both $H$. pylori-infected mucosa and intestinal metaplasia. Haematoxylin and eosin slides were reassessed by a consultant pathologist (SP) to ensure correct classification. All cases of $H$. pylori-associated gastritis showed significant numbers of organisms. The epithelial dysplasia group was classified as low- $(n=6)$ or high- $(n=6)$ grade. Intestinal metaplasia was present in 6 of the 12 dysplasia biopsies.
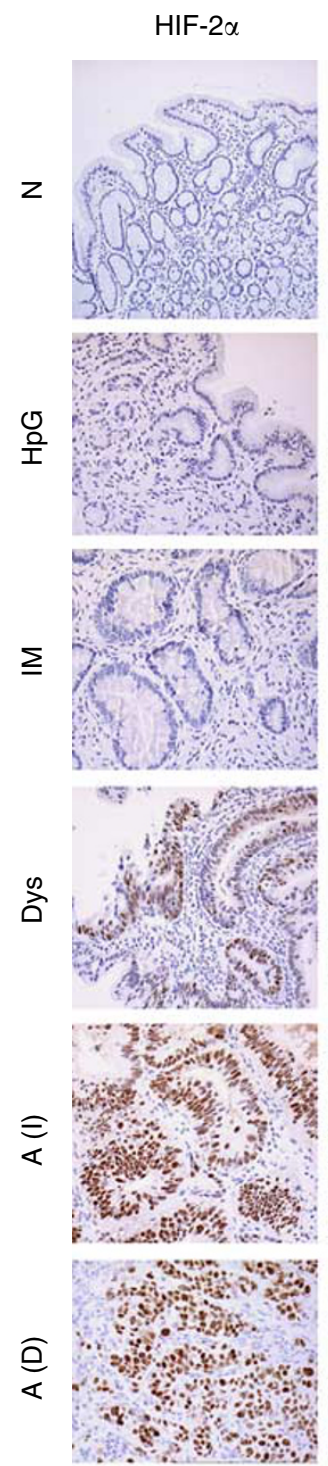

VEGF
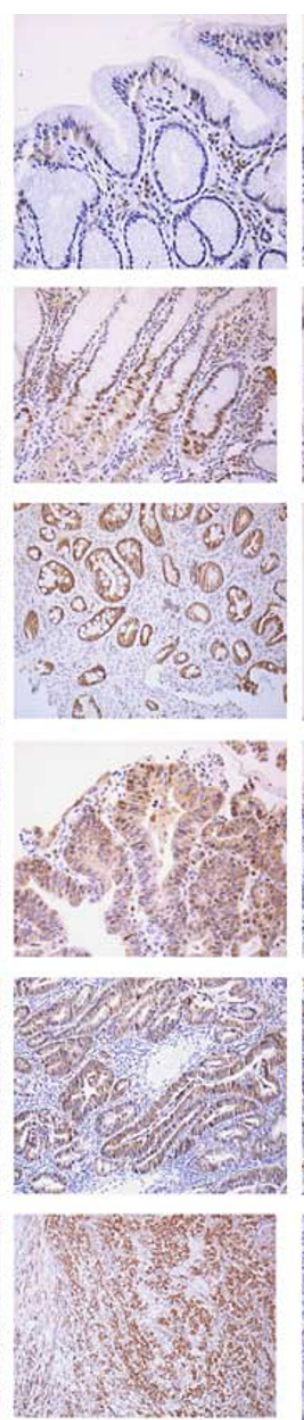

Epo
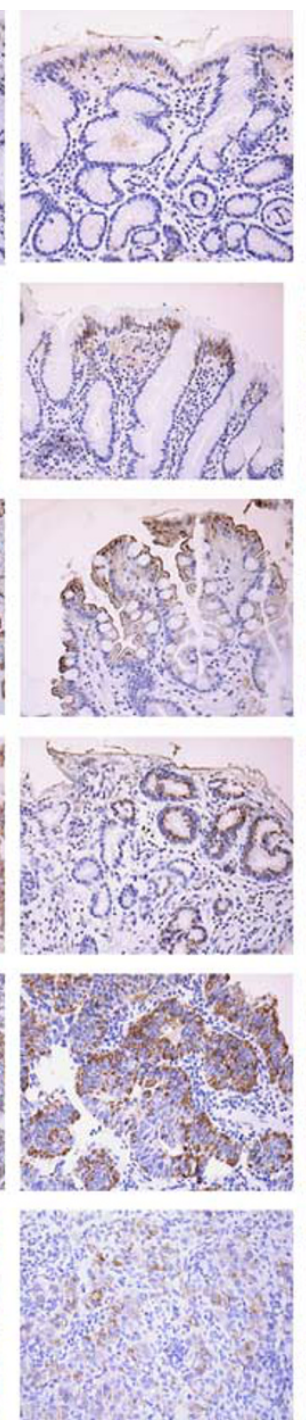

Epo-R
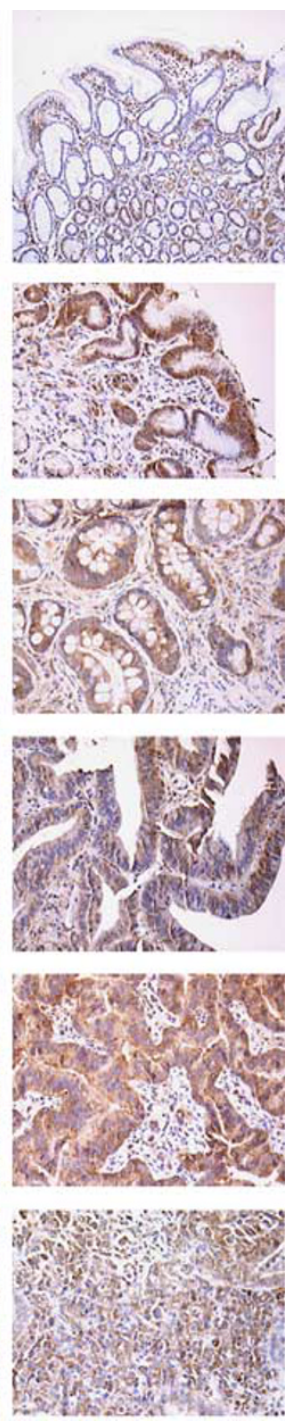

Figure I Representative photomicrographs of HIF-2 $\alpha$, VEGF, Epo, Epo-R immunohistochemistry in the gastric cancer progression sequence. $A(I)=$ intestinal adenocarcinoma; $A(D)=$ diffuse adenocarcinoma; Dys = dysplasia; Epo = erythropoietin; Epo-R=erythropoietin receptor; HIF-2 $\alpha=$ hypoxia-inducible factor- $2 \alpha ; \mathrm{HpG}=H$. pylori gastritis; $I M=$ intestinal metaplasia; $N=$ normal mucosa; VEGF= vascular endothelial growth factor. 


\section{Surgically treated patients}

A retrospectively compiled database was established of 251 consecutive patients with primary gastric and gastro-oesophageal junction tumours who underwent surgery at the South Manchester University Hospitals NHS Trust between 1995 and 2004. The Siewert classification was used to classify gastro-oesophageal junction tumours (Siewert and Stein, 1998). Patients who had either Siewert type I gastro-oesophageal tumours $(n=22)$, neoadjuvant therapy $(n=31)$, emergency surgery $(n=1)$, completion gastrectomy $(n=6)$ or died after surgery $(n=25)$ were excluded from the study. The study group therefore comprised 177 patients (125 men) with a median age of 68 (range 49-85) years. There were 76 Siewert type II, 21 type III gastro-oesophageal junction tumours and 80 noncardia gastric cancers. Patients underwent either partial or subtotal gastrectomies $(n=45)$, total gastrectomy $(n=44)$, proximal gastrectomy $(n=4)$ or oesophago-gastrectomy $(n=84)$. Selected patients underwent additional surgical resection of the spleen $(n=21)$ and spleen with distal pancreas $(n=5)$. One hundred and thirteen patients (64\%) underwent a potentially curative resection (R0). Fifty-four patients $(31 \%)$ had residual microscopic disease (R1 resection) and 10 patients $(6 \%)$ had residual macroscopic disease ( $\mathrm{R} 2$ resection). After surgery, patients were followed in the surgical outpatient clinic. Hospital notes of the patients were reviewed and, if necessary, the local cancer registry or patient's general practitioner was contacted to complete case follow-up.

\section{Immunohistochemistry}

Antigen retrieval was carried out by microwaving for $25 \mathrm{~min}$ in either $10 \mathrm{~mm}$ sodium citrate ( $\mathrm{pH} 6.0$ ) or $0.05 \mathrm{M}$ Tris- $\mathrm{HCl}$ (SigmaAldrich Ltd., Poole, UK)/1 mm EDTA (Sigma) (pH 8.5 or 9.0) buffer solution. After quenching endogenous peroxidase, nonspecific binding was blocked using $10 \%$ casein solution (Vector Laboratories Ltd., Peterborough, UK). The primary antibody was applied and the sections incubated as described elsewhere (Griffiths et al, 2007a). Mouse or rabbit EnVisionPlus System (Dako, UK) was used for antigen detection. Identical concentrations of immunoglobulin IgG1 (Dako Ltd., Ely, UK) from the same species were used as negative controls. Positive and negative (or low) tissue controls from gastric, cervical or head and neck cancer with known staining characteristics were used in each batch.
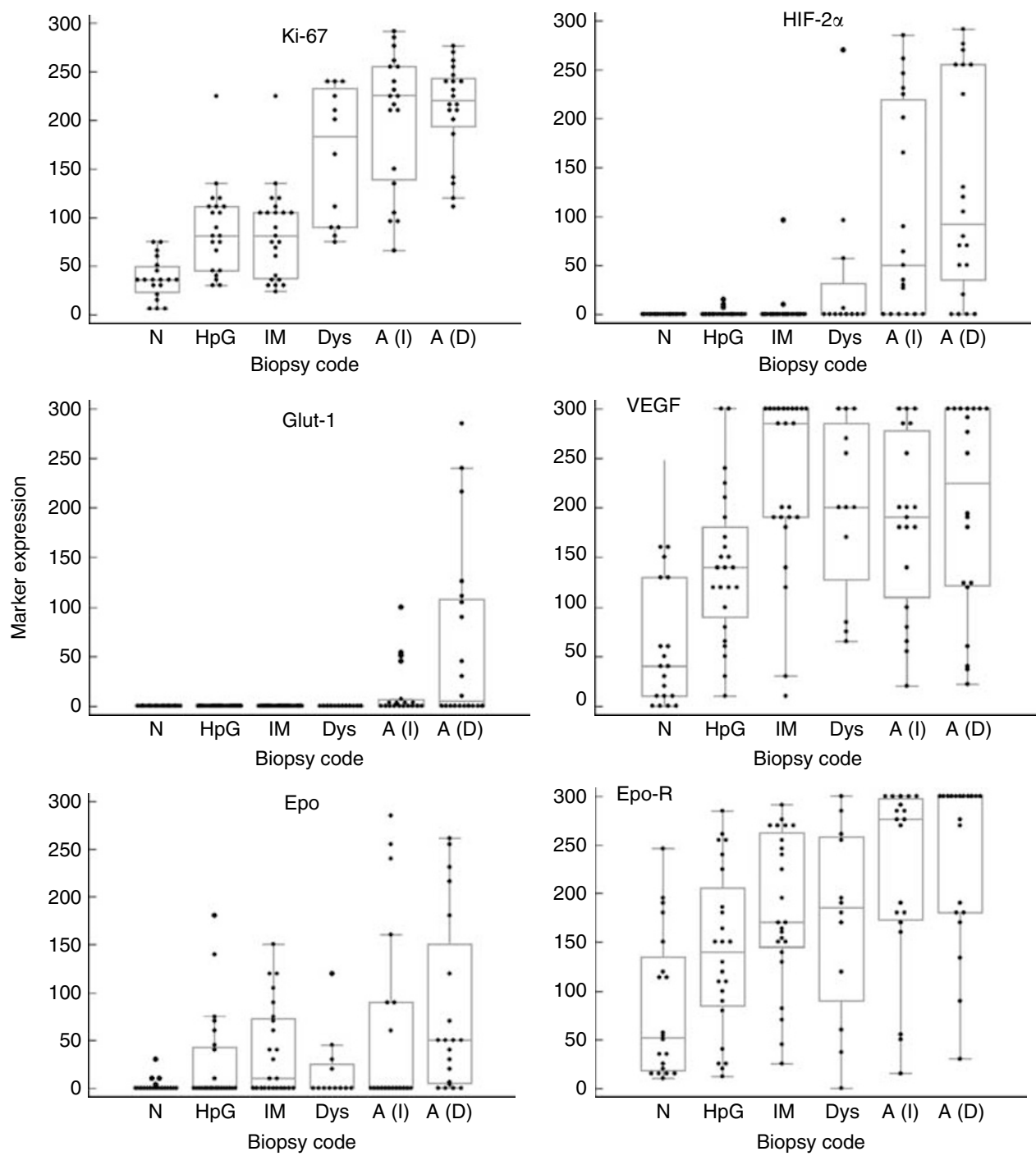

Figure 2 Box and whisker plots of each immunohistochemical marker in the gastric carcinogenesis sequence. The box represents $25-75$ quartiles with a median line. The whiskers extend to minimum and maximum values, but exclude outlying and far-out values. $A(I)=$ intestinal adenocarcinoma; $\mathrm{A}(\mathrm{D})=$ diffuse adenocarcinoma; Dys = dysplasia; Epo = erythropoietin; Epo-R = erythropoietin receptor; HIF-2 $\alpha=$ hypoxia-inducible factor-2 $\alpha$; HpG = $H$. pylori gastritis; $I M=$ intestinal metaplasia; $N=$ normal mucosa; $V E G F=$ vascular endothelial growth factor. 
Batch-to-batch variation was assessed by running sections showing high and low protein expression with each batch. Antibodies were visualised with 3,3'-diaminobenzidine (Dako) and the sections lightly counterstained with haematoxylin, dehydrated and coverslipped.

\section{Scoring}

Scoring was performed in a double-blind manner by two investigators (SAP, SMG). Any disagreement was resolved by discussion to obtain final scores. Markers (HIF-2 $\alpha$, VEGF, EPO, EPO-R, Glut-1, and Ki-67) in the carcinogenesis study were scored using the same scoring system. A score $(0-300)$ was calculated for each marker by multiplying intensity (none 0 , weak 1 , moderate 2 , strong 3) with percentage of expression (range 0-100). For the prognostic study, tumour nuclear HIF- $1 \alpha$ and HIF- $2 \alpha$ staining was scored as follows: 0 , no staining; $1,<2 \%$ staining; $2,2-10 \%$ staining; 3, 11-29\% staining; and 4, >30\% staining. Hypoxiainducible factor- $1 \alpha$ scores were obtained from our previous research (Griffiths et al, 2007b).

\section{Statistics}

Data were analysed using SPSS version 11.5. The Spearman's rank test was used to investigate relationships between variables. Differences in expression levels in the carcinogenesis studies were assessed using Mann-Whitney and Kruskal-Wallis tests. The Jonckheere-Terpstra test was used to identify ordered differences in marker expression. With this test, the null hypothesis is that distributions do not differ across ordered categories. The $\chi^{2}$ test was used to correlate tumour HIF- $2 \alpha$ expression with clinicopathological characteristics.

Survival time was measured as the time from the date of surgery until death or last follow-up appointment. Overall and cancerspecific survival were used as end points. At the time of analysis, 51 patients were alive with a median follow-up of 48 (range 13118) months and 107 had died of disease with a median time to death of 14 (range 2-74) months. There were 16 intercurrent deaths from other causes. Univariate survival analyses were illustrated using the Kaplan-Meier method. Factors were compared using the Cox proportional hazards model and log-rank tests. Multivariate survival analysis was performed on factors that achieved statistical significance in univariate analysis, using the Cox proportional hazards model to identify independent predictors of survival. All statistical tests were two-sided at the 0.05 significance level. As adjusting statistical significance depending on the number of tests performed can create problems (Perneger, 1998), no allowance was made for multiple testing.

\section{RESULTS}

\section{Staining}

Figure 1 shows photomicrographs of HIF-2 $\alpha$, VEGF, Epo and Epo-R staining in the gastric cancer progression sequence. Only

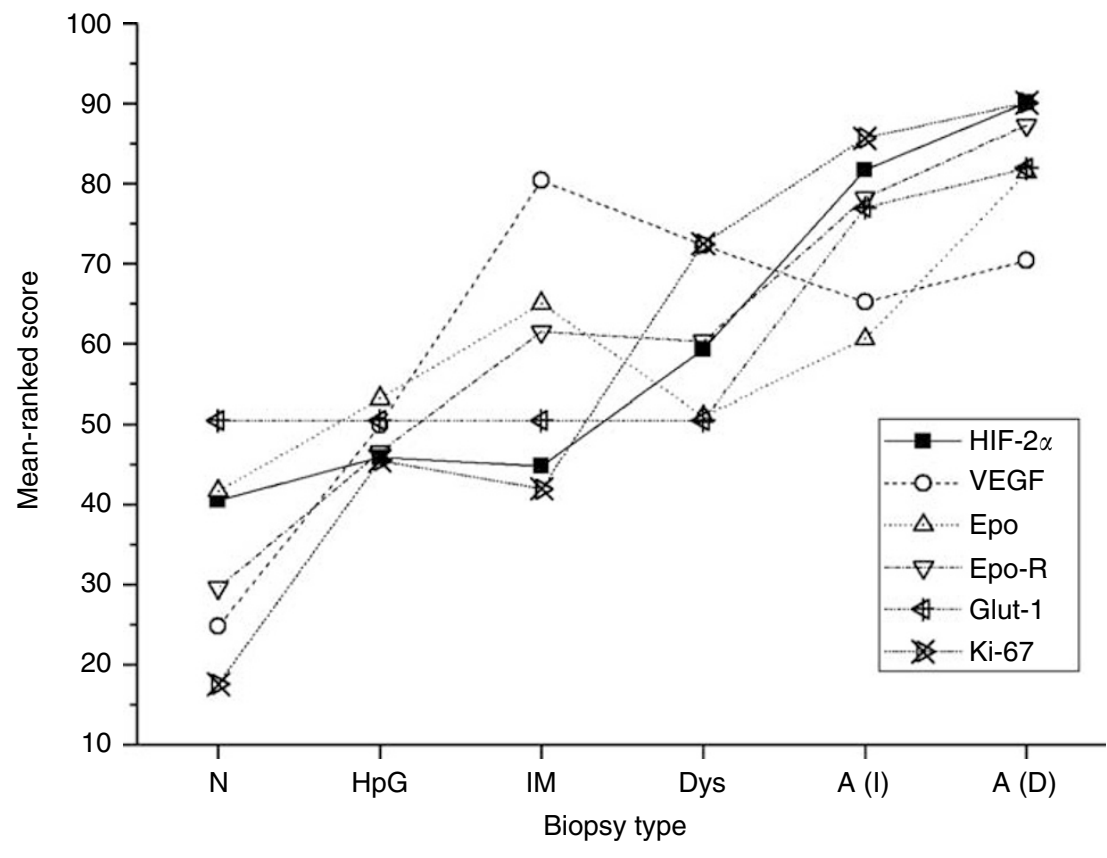

\begin{tabular}{lcccccccc}
\hline & \multicolumn{4}{c}{ Percentiles } & \multicolumn{2}{c}{ Kruskall-Wallis } & \multicolumn{2}{c}{ Jonckheere-Terpstra } \\
Marker & $\boldsymbol{n}$ & 25th & 50th & 75th & $\chi^{2}$ & $\boldsymbol{P}$ & Statistic & $\boldsymbol{P}$ \\
\hline HIF-2 $\alpha$ & $118^{*}$ & 0 & 0 & 38.75 & 56.2 & 0.0001 & 6.9 & 0.0001 \\
VEGF & $119^{*}$ & 75 & 180 & 285 & 35.4 & 0.0001 & 4.6 & 0.0001 \\
Epo & $118^{*}$ & 0 & 0 & 50 & 18.8 & 0.02 & 3.6 & 0.0001 \\
Epo-R & $119^{*}$ & 90 & 180 & 270 & 37.2 & 0.0001 & 6.4 & 0.0001 \\
Glut-1 & $119^{*}$ & 0 & 0 & 0 & 46.0 & 0.0001 & 5.8 & 0.0001 \\
Ki-67 & $114^{*}$ & 57.75 & 105 & 216 & 71.4 & 0.0001 & 8.6 & 0.0001 \\
\hline
\end{tabular}

Figure 3 The mean rank score (Kruskall-Wallis test) of each marker in relation to the gastric carcinogenesis sequence. Immunohistochemical score was calculated from percentage $(0-100)$ multiplied by intensity $(0-3)$ of expression for each marker studied. *Insufficient biopsy tissue for marker scoring in some sections. The Kruskall-Wallis and Jonckheere-Terpstra test results for each immunohistochemical marker studied in the gastric carcinogenesis sequence. $A(I)=$ intestinal adenocarcinoma; $A(D)=$ diffuse adenocarcinoma; Dys = dysplasia; Epo=erythropoietin; Epo- $R=$ erythropoietin receptor; HIF- $2 \alpha=$ hypoxia-inducible factor- $2 \alpha ; \mathrm{HpG}=H$. pylori gastritis; IM = intestinal metaplasia; $N=$ normal mucosa; VEGF= vascular endothelial growth factor. 
nuclear HIF-2 $\alpha$ staining was scored, however, occasional cytoplasmic staining was seen. Hypoxia-inducible factor- $2 \alpha$ was not expressed in normal tissue. Low levels of expression were found in H. pylori gastritis and intestinal metaplasia biopsies. Vascular endothelial growth factor staining was cytoplasmic. Normal gastric mucosa showed weak staining mainly in the deep portions of the crypts that tended to be basal in location, in cytoplasm around the nucleus. Areas of intestinal metaplasia showed increased staining compared with normal mucosa and adjacent nonmetaplastic mucosa within the same biopsy. Erythropoietin staining was cytoplasmic and predominantly focal in nature; Epo- $R$ was expressed in cytoplasm and membrane. Inflammatory cells acted as an internal positive control. Glut-1 staining was cytoplasmic and/or membranous, and detected only in invasive cancer biopsies. In normal gastric mucosa, nuclear Ki-67 staining was present mainly in the cells in the neck region of the gastric pits. In neoplastic tissue, this normal staining pattern was lost and more diffuse expression seen. In all cases along the gastric carcinogenesis sequence, when specialised gastric body-type cells (chief and parietal cells) were present in the biopsy, they showed intense staining for VEGF and Epo-R in a uniform fashion. As the staining was identical in all cases these areas were not scored.

\section{Expression of markers along the gastric carcinogenesis sequence}

Interobserver agreement for marker scores was highly statistically significant and consistent for all the markers studied $(P<0.0001$ for all). With the exception of Glut-1 and VEGF, there were statistically significant correlations between the expression levels of the various markers. For example, HIF- $2 \alpha$ expression correlated with VEGF $(r=0.20, P=0.03)$, Epo $(r=0.34, P<0.001)$, Epo-R $(r=0.46, P<0.001)$, Glut-1 $(r=0.38, P<0.001)$, Ki-67 $(r=0.59$, $P=0.59, P<0.001)$ and also HIF- $1 \alpha(r=0.34, P<0.001)$. Box and whisker plots (including individual data points) are shown in Figure 2 and mean rank scores (from the Kruskall-Wallis test statistics) are plotted in Figure 3. There was a statistically significant increase in the expression of all markers along the progression sequence to adenocarcinoma.

\section{Expression of HIF-2 $\alpha$ in surgically resected specimens}

Tumours tended to show diffuse staining for HIF- $2 \alpha$ in almost all nuclei or negative staining (Figure 1). There was no obvious association with inflammation, ulceration or infiltrative edge and location of HIF- $2 \alpha$ positive staining. In nine slides, cytoplasmic staining was present; this was not scored. Focal staining was identified in some inflammatory cells that acted as an internal positive control. Five sections $(2.8 \%)$ had insufficient tissue for HIF- $2 \alpha$ scoring. In 66 sections (37.3\%), no HIF- $2 \alpha$ immunostaining was observed. Positive nuclear staining was as follows: $<2 \%$ staining in 9 sections (5.1\%), $2-10 \%$ staining in 13 sections $(7.3 \%), 11-30 \%$ staining in 6 sections $(3.4 \%)$ and $>30 \%$ staining in 78 sections $(44.1 \%)$. All negative controls showed no immunoreactivity. No statistically significant relationships were found between the expression of HIF- $1 \alpha$ (Griffiths et al, 2007b) and HIF- $2 \alpha(P=0.31)$.

\section{HIF- $2 \alpha$ expression and clinicopathological features}

For correlation with various clinicopathological features, HIF- $2 \alpha$ expression was categorised as negative (score 0 ) and positive (scores 1/2/3/4). The distribution of patients according to tumour HIF- $2 \alpha$ expression is shown in Table 1 . Hypoxia-inducible factor$2 \alpha$-positive tumours were more likely to be diffuse $(P=0.025)$. There was also a trend for HIF- $2 \alpha$ tumours to have a more advanced $\mathrm{T}$ stage $(P=0.058)$. No statistically significant correlations were found between HIF- $2 \alpha$ and differentiation, N stage, $\mathrm{M}$ stage, overall TNM stage or R classification.

\section{HIF- $2 \alpha$ expression and patient survival}

Tumour HIF- $2 \alpha$ expression was a statistically significant adverse prognostic factor (Figure 4 and Table 2). The median overall survival for patients with tumour HIF- $2 \alpha$ expression was $22(95 \%$ CI 18-26) months, whereas HIF-2 $\alpha$-negative patients had a median survival of $37(95 \%$ CI $29-44)$ months $(P=0.015)$. Hypoxia-inducible factor- $2 \alpha$ expression was more prognostic for gastric $(P=0.032)$ than gastro-oesophageal $(P=0.26)$ tumours (Figure 4). Other significant factors in univariate survival analyses were tumour differentiation, $\mathrm{T}$ stage, $\mathrm{N}$ stage, overall TNM stage and $\mathrm{R}$ classification (Table 2 ).

The combined effect of tumour HIF- $1 \alpha$ (Griffiths et al, 2007b) and HIF- $2 \alpha$ expression was analysed in relation to patient outcome. Patients with invasive edge HIF- $1 \alpha$ - and HIF- $2 \alpha-$ expressing tumours had a poorer overall survival than those with HIF- $1 \alpha$-negative/focally positive and HIF- $2 \alpha$-negative cancers $(P=0.006)$. The median overall survival times were 17 (95\% CI $4-30)$ and 40 (95\% CI 32-42) months, respectively. Corresponding figures for cancer-specific survival were 41 and 17 (95\% CI $4-30)$ months $(P=0.007)$. As for HIF- $1 \alpha$ (Griffiths et al, 2007b) and HIF- $\alpha$ (Figure 4) alone, the combined adverse effect of invasive edge HIF- $1 \alpha$ and HIF- $2 \alpha$ expression was greater for gastric

Table I The distribution of patient characteristics according to tumour expression of HIF- $2 \alpha(n=172)$

\begin{tabular}{|c|c|c|c|}
\hline Factor & HIF- $2 \alpha$ negative & HIF- $2 \alpha$ positive & $\mathbf{P}^{*}$ \\
\hline \multicolumn{4}{|c|}{ Differentiation } \\
\hline Well & 8 & 10 & \\
\hline Mod & 24 & 41 & \\
\hline Poor & 34 & 55 & 0.84 \\
\hline \multicolumn{4}{|l|}{ Lauren type } \\
\hline Diffuse & 27 & 62 & \\
\hline Intestinal & 39 & 44 & 0.025 \\
\hline \multicolumn{4}{|l|}{ T stage } \\
\hline T in-situ & 0 & 3 & \\
\hline $\mathrm{TI}$ & 7 & 9 & \\
\hline $\mathrm{T} 2$ & 27 & 26 & \\
\hline T3 & 32 & 64 & \\
\hline $\mathrm{T} 4$ & 0 & 4 & 0.058 \\
\hline \multicolumn{4}{|l|}{$N$ stage } \\
\hline No & 20 & 29 & \\
\hline $\mathrm{NI}$ & 37 & 62 & \\
\hline N2 & 7 & 13 & \\
\hline N3 & 2 & 2 & 0.921 \\
\hline \multicolumn{4}{|l|}{ M stage } \\
\hline $\mathrm{MO}^{\circ}$ & 65 & 103 & \\
\hline MI & I & 3 & 0.58 \\
\hline \multicolumn{4}{|c|}{ Overall TNM stage } \\
\hline 0 & 0 & 3 & \\
\hline 1 & 14 & 15 & \\
\hline$\|$ & 23 & 31 & \\
\hline III & 27 & 50 & \\
\hline IV & 2 & 7 & 0.31 \\
\hline \multicolumn{4}{|l|}{$R$ class } \\
\hline RO & 46 & 64 & \\
\hline RI & 19 & 33 & \\
\hline $\mathrm{R} 2$ & I & 9 & 0.13 \\
\hline
\end{tabular}

$\mathrm{HIF}=$ hypoxia-inducible factor. $* \chi^{2} P$-value 

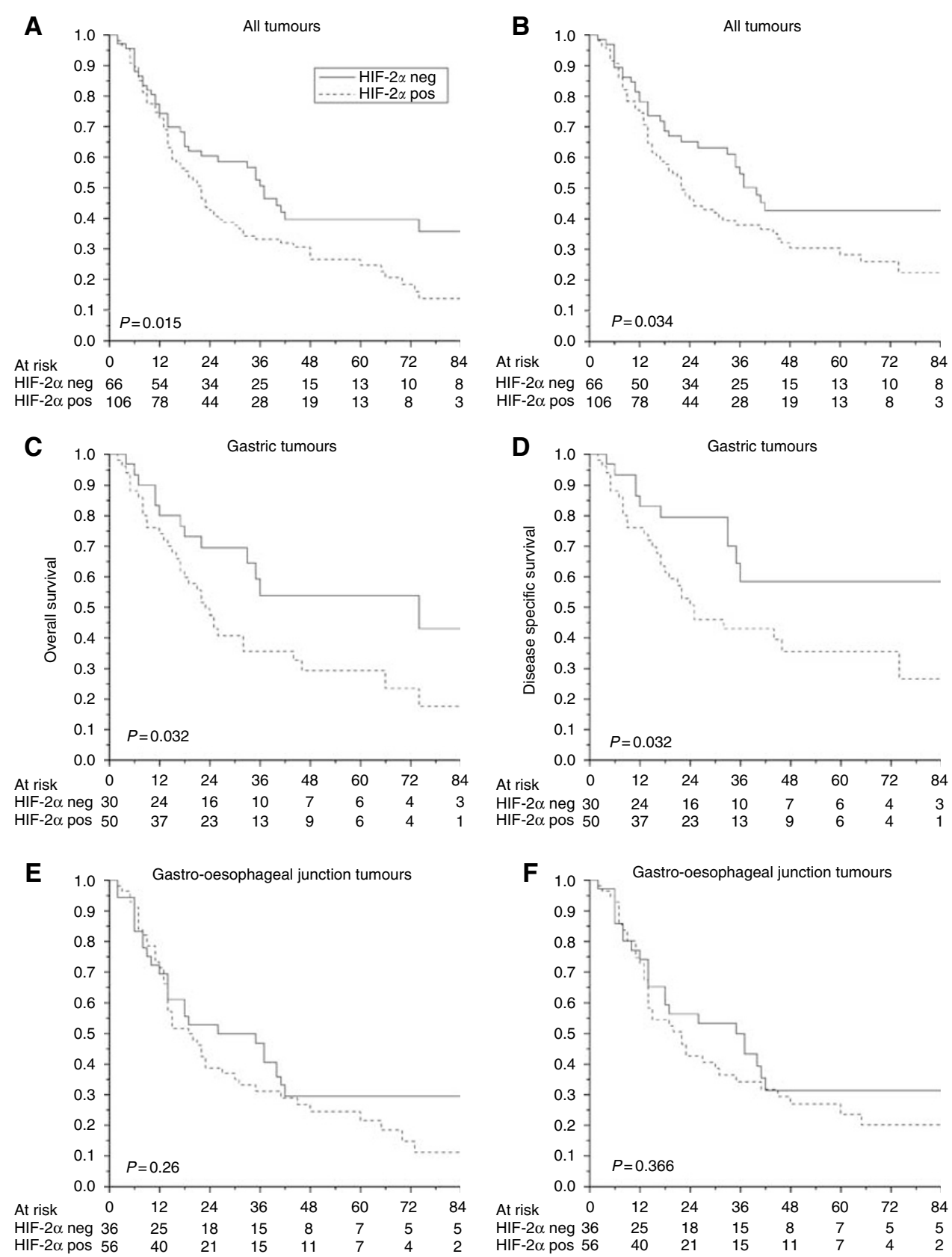

Figure 4 Hypoxia-inducible factor- $2 \alpha$ expression and patient outcome in 172 tumours including noncardia gastric cancers $(n=80)$ and gastrooesophageal junction tumours $(n=92)$. The first column shows HIF- $2 \alpha$-negative (score 0 ) vs positive (scores I/2/3/4) expression and overall survival. For all tumours $(\mathbf{A})$, gastric cancers $(\mathbf{C})$ and gastro-oesophageal junction tumours $(\mathbf{E})$. Second column shows disease-specific survival. For all tumours $(\mathbf{B})$, gastric tumours (D) and gastro-oesophageal junction tumours (F). HIF = hypoxia-inducible factor.

than for gastro-oesophageal junction tumours for both diseasespecific $(P=0.026$ vs 0.104$)$ and overall $(P=0.047$ vs 0.089$)$ survival (Figure 5). For example, patients with HIF-1 $\alpha$-negative/ focally positive and HIF- $2 \alpha$-negative gastric cancers had an average disease-free survival of 81 (95\% CI 59-103) months compared with 25 (95\% CI 11-39) months for those with invasive edge HIF- $1 \alpha$ - and HIF- $2 \alpha$-positive tumours. All analyses were repeated stratifying patients by $0 / 1$ vs $2 / 3 / 4$ scores. Although qualitatively similar results were seen, they were not statistically significant.

All factors that achieved statistical significance $(P<0.05)$ in the univariate analysis were entered into a multivariate analysis. Neither HIF- $2 \alpha$ expression nor HIF- $1 \alpha /$ HIF- $2 \alpha$ combined had any independent prognostic significance. Only tumour differentiation, overall TNM stage and $\mathrm{R}$ classification retained significance in multivariate analysis.

\section{DISCUSSION}

All markers studied showed a statistically significant increase in expression with progression from $H$. pylori-associated gastritis, intestinal metaplasia, dysplasia to adenocarcinoma $(P=0.0001)$. It must be emphasised, however, that this model does not apply to gastro-oesophageal junction tumours. Gastro-oesophageal adenocarcinomas (Siewert type I and II tumours) arise via a similar sequence of histopathological events; however, the initiating, 
Table 2 Univariate survival analyses

\begin{tabular}{|c|c|c|c|c|c|c|}
\hline \multirow[b]{2}{*}{ Parameter } & \multicolumn{3}{|c|}{$\begin{array}{l}\text { Overall } \\
\text { survival }\end{array}$} & \multicolumn{3}{|c|}{$\begin{array}{l}\text { Disease-specific } \\
\text { survival }\end{array}$} \\
\hline & HR & $95 \% \mathrm{Cl}$ & $P^{*}$ & HR & $95 \% \mathrm{Cl}$ & $\boldsymbol{P}^{*}$ \\
\hline \multicolumn{7}{|l|}{$H I F-\mid \alpha$} \\
\hline 0 & I & - & - & I & - & \\
\hline $1 / 2 / 3 / 4$ & 1.1 & $0.8-1.4$ & 0.62 & 1.0 & $0.7-1.5$ & 0.82 \\
\hline Negative & I & - & - & I & - & - \\
\hline Focal & 0.9 & $0.5-1.3$ & 0.49 & 0.7 & $0.5-1.2$ & 0.26 \\
\hline Invasive edge & 1.6 & $1.0-2.4$ & 0.042 & 1.6 & $1.0-2.5$ & 0.047 \\
\hline \multicolumn{7}{|l|}{$H I F-2 \alpha$} \\
\hline 0 & 1.0 & - & - & 1.0 & - & - \\
\hline $1 / 2 / 3 / 4$ & 1.6 & $1.1-2.4$ & 0.018 & 1.6 & $1.0-2.4$ & 0.038 \\
\hline \multicolumn{7}{|l|}{$H I F-\mid \alpha / H I F-2 \alpha$} \\
\hline HIF- $\mid \alpha$ neg/HIF-2 $\alpha$ neg & 1.0 & - & - & 1.0 & - & - \\
\hline HIF- I $\alpha$ pos/HIF- $2 \alpha$ neg & 1.5 & $0.8-2.9$ & 0.224 & I.I & $0.6-2.2$ & 0.76 \\
\hline HIF- $\mid \alpha$ neg/HIF- $2 \alpha$ pos & 2.0 & $1.2-3.6$ & 0.014 & 1.6 & $0.9-3.0$ & 0.095 \\
\hline HIF-I $\alpha$ pos/HIF- $2 \alpha$ pos & 1.8 & $1.1-3.3$ & 0.036 & 1.6 & $0.9-2.9$ & 0.12 \\
\hline \multicolumn{7}{|l|}{$H I F-\mid \alpha / H I F-2 \alpha$} \\
\hline HIF- $2 \alpha$ neg/HIF-I $\alpha$ neg/focal & 1.0 & - & - & 1.0 & - & - \\
\hline $\mathrm{HIF}-2 \alpha$ neg/HIF-I $\alpha$ inv & 2.1 & $1.0-4.3$ & 0.37 & 1.7 & $0.7-3.7$ & 0.22 \\
\hline HIF- $2 \alpha$ pos/HIF-I $\alpha$ neg/focal & 1.8 & $1.1-2.8$ & 0.16 & 1.5 & $0.9-2.5$ & 0.10 \\
\hline HIF- $2 \alpha$ pos/HIF- $\alpha$ inv & 2.6 & $1.5-4.6$ & 0.001 & 2.7 & $1.5-4.8$ & 0.001 \\
\hline \multicolumn{7}{|l|}{ Diff } \\
\hline Well & 1.0 & - & - & 1.0 & - & - \\
\hline Mod & 2.9 & $1.4-6.2$ & 0.005 & 3.4 & $1.3-8.5$ & 0.011 \\
\hline Poor & 3.7 & $1.8-7.8$ & 0.001 & 5.3 & $2.1-13.3$ & 0.001 \\
\hline \multicolumn{7}{|l|}{ Lauren type } \\
\hline Intestinal & 1.0 & - & - & 1.0 & - & - \\
\hline Diffuse & 1.4 & $1.0-2.0$ & 0.052 & 1.8 & $1.2-2.6$ & 0.003 \\
\hline \multicolumn{7}{|l|}{ Location } \\
\hline Non-GOJ & 1.0 & - & - & 1.0 & - & - \\
\hline GOJ & 1.4 & $1.0-2.0$ & 0.083 & 1.5 & $1.0-2.2$ & 0.059 \\
\hline \multicolumn{7}{|l|}{ T stage } \\
\hline TO/I & 1.0 & - & - & 1.0 & - & - \\
\hline T2 & 2.6 & $1.0-6.7$ & 0.052 & 5.2 & $1.2-22.0$ & 0.023 \\
\hline T3 & 4.8 & $1.9-12.0$ & 0.001 & 9.6 & $2.3-39.0$ & 0.002 \\
\hline T4 & 16.8 & $4.4-64.2$ & 0.0001 & 37.5 & $6.8-207.6$ & 0.0001 \\
\hline \multicolumn{7}{|l|}{$N$ stage } \\
\hline No & 1.0 & - & - & 1.0 & - & - \\
\hline $\mathrm{NI}$ & 2.0 & $1.3-3.0$ & 0.003 & 2.5 & $1.5-4.1$ & 0.001 \\
\hline N2 & 3.5 & $1.9-6.4$ & 0.0001 & 4.8 & $2.5-9.2$ & 0.0001 \\
\hline N3 & 4.2 & $1.5-12.0$ & 0.008 & 5.7 & $1.9-16.9$ & 0.002 \\
\hline \multicolumn{7}{|l|}{ M stage } \\
\hline MO & 1.0 & - & - & I & - & - \\
\hline MI & 2.6 & $1.0-7.1$ & 0.062 & 2.9 & $1.1-7.9$ & 0.037 \\
\hline \multicolumn{7}{|l|}{ Overall TNM stage } \\
\hline $0 / 1$ & 1.0 & - & - & 1.0 & - & - \\
\hline 2 & 1.4 & $0.8-2.6$ & 0.25 & 1.8 & $0.9-3.6$ & 0.12 \\
\hline 3 & 3.3 & $1.9-5.9$ & 0.0001 & 4.5 & $2.3-8.8$ & 0.0001 \\
\hline 4 & 7.6 & $3.3-17.5$ & 0.0001 & 10.9 & $4.4-27.1$ & 0.0001 \\
\hline \multicolumn{7}{|l|}{$R$ class } \\
\hline RO & 1.0 & - & - & 1.0 & - & - \\
\hline RI & 2.3 & $1.6-3.3$ & 0.0001 & 2.7 & $1.8-4.0$ & 0.0001 \\
\hline R2 & 5.8 & $2.9-11.6$ & 0.0001 & 7.2 & $3.6-14.5$ & 0.0001 \\
\hline
\end{tabular}

$\mathrm{Cl}=$ confidence interval; diff=differentiation; $\mathrm{GEJ}=$ gastro-oesophageal junction; $\mathrm{HIF}=$ hypoxia-inducible factor; $\mathrm{HR}=$ hazard ratio. ${ }^{*} P$-value from a univariate Coxproportional hazards model. HIF-I $\alpha$ results obtained from Griffiths et al (2007b). promoting and molecular factors are different to gastric cancer carcinogenesis. Indeed, $H$. pylori appears to exert a protective role in these types of tumours (Chow et al, 1998). We have previously assessed these immunohistochemical markers in a similar model of oesophageal carcinogenesis (Griffiths et al, 2007a).

Previous studies have shown the importance of Ki-67 expression and proliferation in the progression of gastric cancer. For example, Leung et al (2001) showed that proliferation, as measured by Ki-67 expression, was significantly increased in both $H$. pylori-infected and intestinal metaplasia biopsy material. Interestingly, they also found that the apoptotic to proliferation index ratio was significantly reduced, favouring cellular DNA-damage accumulation and neoplastic progression. Similarly, the role of VEGF and angiogenesis in the gastric carcinogenesis sequence has been previously reported. We found similar results to that of Feng et al (2002), who assessed VEGF expressed by immunohistochemistry. Our finding that Glut-1 protein is not found in normal gastric mucosa but only expressed in gastric carcinoma also confirms the results of others (Younes et al, 1996; Kim et al, 2000; Kawamura et al, 2001). For example, one study reported no expression of Glut-1 in normal gastric mucosa, intestinal metaplasia and tubular adenomas but $30 \%$ of 617 gastric carcinomas was positive for the protein (Kawamura et al, 2001).

Erythropoietin and Epo-R have been shown to be expressed in a number of cancers and involved in breast (Acs et al, 2002), endometrial (Acs et al, 2004), melanoma (Kumar et al, 2005), prostate (Feldman et al, 2006) and Barrett's oesophageal (Griffiths et al, 2007a) carcinogenesis. There appear to be no other studies that have assessed these proteins in gastric carcinogenesis. Both Epo and Epo-R were expressed in varying degrees in all biopsy types, but Epo-R was expressed more abundantly. The expression of both proteins increased from normal tissue through to invasive cancer. Endogenous Epo is a glycoprotein produced primarily in the adult kidney in response to hypoxia. Its main function is to regulate erythropoiesis by stimulating growth, inhibiting apoptosis and inducing differentiation of red blood cell precursors (Yasuda et al, 2003).

Hypoxia-inducible factor- $2 \alpha$ expression occurred principally as a late phenomenon. However, there was evidence of expression in a minority of the earlier premalignant biopsies of $H$. pylori gastritis and intestinal metaplasia. Hypoxia-inducible factor- $2 \alpha$ expression has not been studied in models of tumour carcinogenesis except in our work in the Barrett's metaplasia-dysplasia-adenocarcinoma sequence, where we showed that HIF- $2 \alpha$ was expressed late in the sequence and was seen only in dysplasia and adenocarcinoma (Griffiths et al, 2007a). Recently, the overexpression of HIF-2 $\alpha$ in rat gliomas was shown to have counteracting properties, in which angiogenesis was induced but reduced growth was also found due to increased tumour-cell apoptosis (Acker et al, 2005). Hypoxiainducible factor $-2 \alpha$ expression has also been suggested to be critical in the carcinogenesis of renal cortical tumours (Kim et al, 2006).

Hypoxia-inducible factor- $2 \alpha$ accumulates in the presence of hypoxia, forms a heterodimer with HIF- $1 \beta$ and binds to hypoxiaresponsive elements. Hypoxia-inducible factor- $2 \alpha$ has been shown to regulate a number of the same hypoxia-inducible genes as HIF- $1 \alpha$ ( $\mathrm{Hu}$ et al, 2003). However, it is now known that the various hypoxia-inducible genes vary in their sensitivity to HIF- $1 \alpha$ and HIF- $2 \alpha$ (Wang et al, 2005) and therefore different downstream pathways can be preferentially activated ( $\mathrm{Hu}$ et al, 2003; Sowter et al, 2003). The classical 'hypoxic' expression of HIF- $2 \alpha$ in tumour sections was not found in our study as there was no association with necrosis or distance from blood vessels. This may suggest nonhypoxic activation. However, unlike HIF- $1 \alpha$, the nonhypoxic activation of HIF- $2 \alpha$ has not been confirmed. A study in breast adenocarcinoma showed a strong correlation between HIF- $2 \alpha$ and c-erbB-2 and suggested that this was due to oncogenic rather than hypoxic activation (Giatromanolaki et al, 2006). To clarify these issues, measurements of the oxygenation status of gastric cancers 

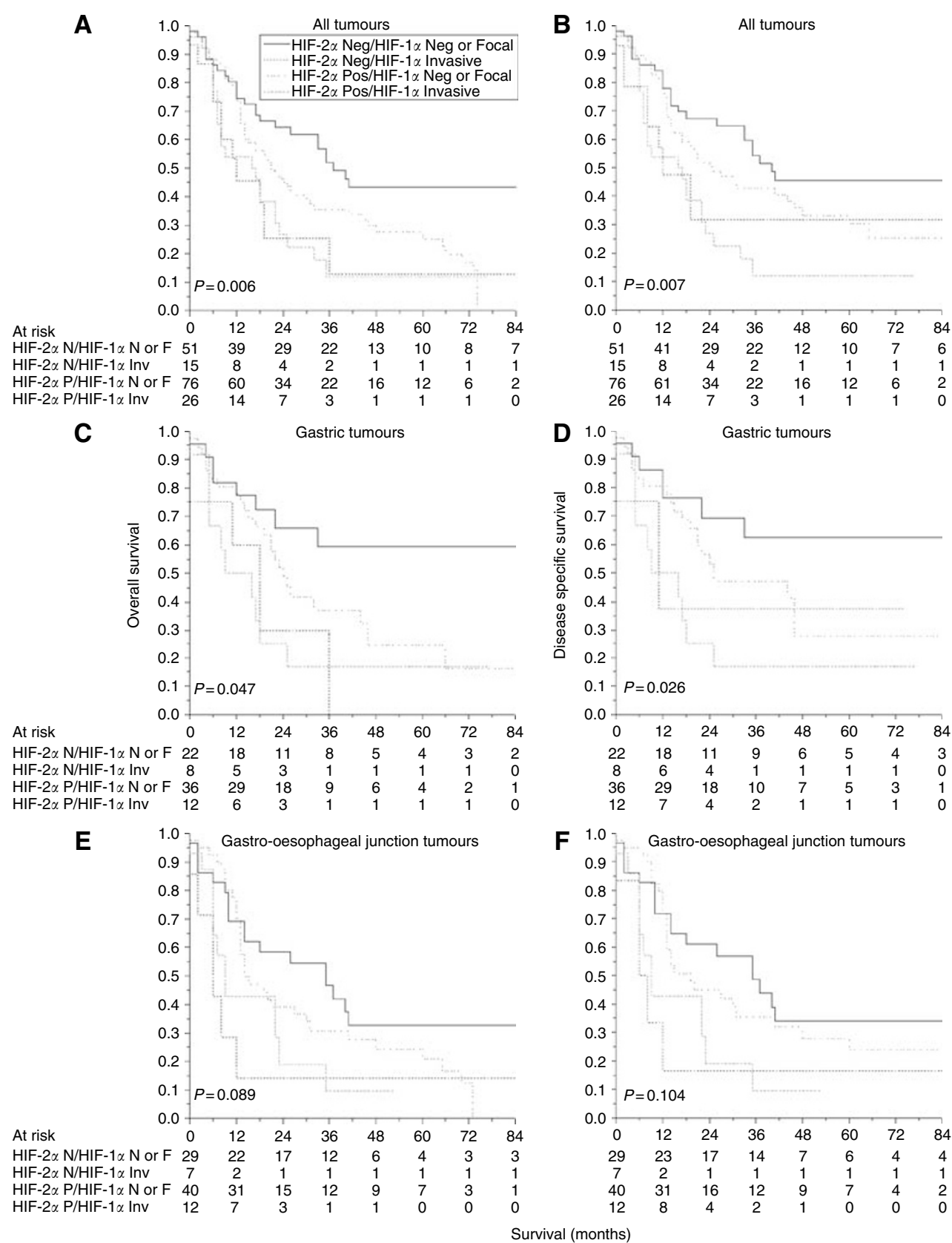

Figure 5 Combined HIF- $\mid \alpha$ and HIF- $2 \alpha$ expression and patient outcome in 168 tumours including noncardia gastric cancers ( $n=78$ ) and gastrooesophageal junction tumours $(n=90)$. The first column shows overall survival $(\mathbf{A}, \mathbf{C}, \mathbf{E})$ and the second column shows disease-specific survival $(\mathbf{B}, \mathbf{D}, \mathbf{F})$. $\mathrm{HIF}=$ hypoxia-inducible factor.

are required (Griffiths et al, 2005). Oxygen electrodes have proved useful in a number of tumour types (Nordsmark et al, 2003), but have limited use in gastric cancer because of poor accessibility. Other approaches such as the immunohistochemical expression of hypoxia-specific markers such as pimonidazole (Nordsmark et al, 2003) and noninvasive imaging (Koch and Evans, 2003) are being developed and could be carried out in patients with gastric cancer.

Few studies have assessed both HIF- $1 \alpha$ and HIF- $2 \alpha$ in relation to patient outcome. Yoshimura et al (2004) examined 87 surgically treated patients with colorectal cancer and found that HIF- $2 \alpha$ but not HIF- $1 \alpha$ expression predicted prognosis in univariate analysis. Other studies in nonsmall cell lung cancer and malignant melanomas showed that HIF-2 $\alpha$ expression was related to a poor outcome when HIF-1 $\alpha$ was not (Giatromanolaki et al, 2001, 2003). These studies confirm the likely tissue-specific differences in the relative importance of HIF proteins in determining tumour progression and prognosis.

The expression of HIF- $2 \alpha$ was significant in univariate analysis; however, it was not an independent predictor of prognosis. In view of the lack of independent prognostic significance, HIF- $2 \alpha$ is unlikely to impact on clinical management. However, the high expression of HIF- $2 \alpha$ in gastric and gastro-oesophageal junction tumours suggests that it may be of value as a potential therapeutic target.

\section{ACKNOWLEDGEMENTS}

The work of the authors is supported by Cancer Research UK and the National Translational Cancer Research Network 
of the UK. We thank David Ryder, Department of Statistics, Christie Hospital and Francesca Buffa, Gray Cancer Institute, Northwood, London, for help with the statistical analyses and Dr Jo Cresswell, Academic Radiation Oncology, Christie Hospital, Manchester for administrative

\section{REFERENCES}

Acker T, Diez-Juan A, Aragones J, Tjwa M, Brusselmans K, Moons L, Fukumura D, Moreno-Murciano MP, Herbert JM, Burger A, Riedel J, Elvert G, Flamme I, Maxwell PH, Collen D, Dewerchin M, Jain RK, Plate $\mathrm{KH}$, Carmeliet P (2005) Genetic evidence for a tumor suppressor role of HIF-2alpha. Cancer Cell 8: 131-141

Acs G, Xu X, Chu C, Acs P, Verma A (2004) Prognostic significance of erythropoietin expression in human endometrial carcinoma. Cancer 100: 2376-2386

Acs G, Zhang PJ, McGrath CM, Acs P, McBroom J, Mohyeldin A, Liu S, Lu $\mathrm{H}$, Verma A (2003) Hypoxia-inducible erythropoietin signaling in squamous dysplasia and squamous cell carcinoma of the uterine cervix and its potential role in cervical carcinogenesis and tumor progression. Am J Pathol 162: 1789 - 1806

Acs G, Zhang PJ, Rebbeck TR, Acs P, Verma A (2002) Immunohistochemical expression of erythropoietin and erythropoietin receptor in breast carcinoma. Cancer 95: 969-981

Bos R, Zhong H, Hanrahan CF, Mommers EC, Semenza GL, Pinedo HM, Abeloff MD, Simons JW, van Diest PJ, van der Wall E (2001) Levels of hypoxia-inducible factor-1 alpha during breast carcinogenesis. J Natl Cancer Inst 93: 309-314

Calzada MJ, del Peso L (2007) Hypoxia-inducible factors and cancer. Clin Transl Oncol 9: 278-289

Chow WH, Blaser MJ, Blot WJ, Gammon MD, Vaughan TL, Risch HA, Perez-Perez GI, Schoenberg JB, Stanford JL, Rotterdam H, West AB, Fraumeni Jr JF (1998) An inverse relation between cagA+ strains of Helicobacter pylori infection and risk of esophageal and gastric cardia adenocarcinoma. Cancer Res 58: 588-590

Correa P (2004) Is gastric cancer preventable? Gut 53: 1217-1219

Costa A, Coradini D, Carrassi A, Erdas R, Sardella A, Daidone MG (2001) Re: levels of hypoxia-inducible factor-1alpha during breast carcinogenesis. J Natl Cancer Inst 93: $1175-1177$

Feldman L, Wang Y, Rhim JS, Bhattacharya N, Loda M, Sytkowski AJ (2006) Erythropoietin stimulates growth and STAT5 phosphorylation in human prostate epithelial and prostate cancer cells. Prostate 66: $135-145$

Feng CW, Wang LD, Jiao LH, Liu B, Zheng S, Xie XJ (2002) Expression of p53, inducible nitric oxide synthase and vascular endothelial growth factor in gastric precancerous and cancerous lesions: correlation with clinical features. BMC Cancer 2: 8

Giatromanolaki A, Koukourakis MI, Sivridis E, Turley H, Talks K, Pezzella F, Gatter KC, Harris AL (2001) Relation of hypoxia inducible factor 1 alpha and 2 alpha in operable non-small cell lung cancer to angiogenic/ molecular profile of tumours and survival. Br J Cancer 85: 881-890

Giatromanolaki A, Sivridis E, Fiska A, Koukourakis MI (2006) Hypoxiainducible factor-2 alpha (HIF-2 alpha) induces angiogenesis in breast carcinomas. Appl Immunohistochem Mol Morphol 14: 78-82

Giatromanolaki A, Sivridis E, Kouskoukis C, Gatter KC, Harris AL, Koukourakis MI (2003) Hypoxia-inducible factors 1alpha and 2alpha are related to vascular endothelial growth factor expression and a poorer prognosis in nodular malignant melanomas of the skin. Melanoma Res 13: $493-501$

Griffiths EA, Pritchard SA, McGrath SM, Valentine HR, Price PM, Welch IM, West CM (2007a) Increasing expression of hypoxia-inducible proteins in the Barrett's metaplasia-dysplasia-adenocarcinoma sequence. Br J Cancer 96: $1377-1383$

Griffiths EA, Pritchard SA, Valentine HR, Whitchelo N, Bishop PW, Ebert MP, Price PM, Welch IM, West CM (2007b) Hypoxia-inducible factorlalpha expression in the gastric carcinogenesis sequence and its prognostic role in gastric and gastro-oesophageal adenocarcinomas. Br J Cancer 96: 95 - 103
Griffiths EA, Pritchard SA, Welch IM, Price PM, West CM (2005) Is the hypoxia-inducible factor pathway important in gastric cancer? Eur J Cancer 41: 2792-2805

Hu CJ, Wang LY, Chodosh LA, Keith B, Simon MC (2003) Differential roles of hypoxia-inducible factor 1alpha (HIF-1alpha) and HIF-2alpha in hypoxic gene regulation. Mol Cell Biol 23: $9361-9374$

Kawamura T, Kusakabe T, Sugino T, Watanabe K, Fukuda T, Nashimoto A, Honma K, Suzuki T (2001) Expression of glucose transporter-1 in human gastric carcinoma: association with tumor aggressiveness, metastasis, and patient survival. Cancer 92: 634-641

Kim CM, Vocke C, Torres-Cabala C, Yang Y, Schmidt L, Walther M, Linehan WM (2006) Expression of hypoxia inducible factor-1alpha and 2alpha in genetically distinct early renal cortical tumors. J Urol 175: $1908-1914$

Kim WS, Kim YY, Jang SJ, Kimm K, Jung MH (2000) Glucose transporter 1 (GLUT1) expression is associated with intestinal type of gastric carcinoma. J Korean Med Sci 15: 420-424

Koch CJ, Evans SM (2003) Non-invasive PET and SPECT imaging of tissue hypoxia using isotopically labeled 2-nitroimidazoles. Adv Exp Med Biol 510: $285-292$

Kumar SM, Acs G, Fang D, Herlyn M, Elder DE, Xu X (2005) Functional erythropoietin autocrine loop in melanoma. Am J Pathol 166: 823-830

Leung WK, Yu J, To KF, Go MY, Ma PK, Chan FK, Sung JJ (2001) Apoptosis and proliferation in Helicobacter pylori-associated gastric intestinal metaplasia. Aliment Pharmacol Ther 15: $1467-1472$

Nardone G, Rocco A, Malfertheiner P (2004) Review article: Helicobacter pylori and molecular events in precancerous gastric lesions. Aliment Pharmacol Ther 20: $261-270$

Nordsmark M, Loncaster J, Aquino-Parsons C, Chou SC, Ladekarl M, Havsteen H, Lindegaard JC, Davidson SE, Varia M, West C, Hunter R, Overgaard J, Raleigh JA (2003) Measurements of hypoxia using pimonidazole and polarographic oxygen-sensitive electrodes in human cervix carcinomas. Radiother Oncol 67: 35-44

Parkin DM, Bray FI, Devesa SS (2001) Cancer burden in the year 2000. The global picture. Eur J Cancer 37(Suppl 8): S4-S66

Perneger TV (1998) What's wrong with Bonferroni adjustments. BMJ 316: $1236-1238$

Ratcliffe PJ (2007) HIF-1 and HIF-2: working alone or together in hypoxia? J Clin Invest 117: $862-865$

Siewert JR, Stein HJ (1998) Classification of adenocarcinoma of the oesophagogastric junction. Br J Surg 85: 1457 - 1459

Sowter HM, Raval RR, Moore JW, Ratcliffe PJ, Harris AL (2003) Predominant role of hypoxia-inducible transcription factor (Hif)-1alpha versus Hif-2alpha in regulation of the transcriptional response to hypoxia. Cancer Res 63: 6130-6134

Wang V, Davis DA, Haque M, Huang LE, Yarchoan R (2005) Differential gene up-regulation by hypoxia-inducible factor-1alpha and hypoxiainducible factor-2alpha in HEK293T cells. Cancer Res 65: 3299-3306

Yasuda Y, Fujita Y, Matsuo T, Koinuma S, Hara S, Tazaki A, Onozaki M, Hashimoto M, Musha T, Ogawa K, Fujita H, Nakamura Y, Shiozaki H, Utsumi H (2003) Erythropoietin regulates tumour growth of human malignancies. Carcinogenesis 24: $1021-1029$

Yoshimura H, Dhar DK, Kohno H, Kubota H, Fujii T, Ueda S, Kinugasa S, Tachibana M, Nagasue N (2004) Prognostic impact of hypoxia-inducible factors 1alpha and 2alpha in colorectal cancer patients: correlation with tumor angiogenesis and cyclooxygenase- 2 expression. Clin Cancer Res 10: $8554-8560$

Younes M, Lechago LV, Somoano JR, Mosharaf M, Lechago J (1996) Wide expression of the human erythrocyte glucose transporter Glut1 in human cancers. Cancer Res 56: $1164-1167$ 